\title{
On particle acceleration and very high energy $\gamma$-ray emission in Crab-like pulsars
}

\author{
Z. Osmanov ${ }^{1}$ and F. M. Rieger ${ }^{2,3}$ \\ ${ }^{1}$ E. Kharadze Georgian National Astrophysical Observatory, Ilia Chavchavadze State University, Kazbegi str. $2 \mathrm{a}, 0106$ Tbilisi, \\ Georgia \\ e-mail: z.osmanov@astro-ge.org \\ 2 Max-Planck-Institut für Kernphysik, Saupfercheckweg 1, 69117 Heidelberg, Germany \\ e-mail: frank.rieger@mpi-hd.mpg.de \\ 3 European Associated Laboratory for Gamma-Ray Astronomy, jointly supported by CNRS and MPG, Germany
}

Received 18 March 2009 / Accepted 18 May 2009

\begin{abstract}
Context. The origin of very energetic charged particles and the production of very high-energy (VHE) gamma-ray emission remains still a challenging issue in modern pulsar physics.

Aims. By applying a toy model, we explore the acceleration of co-rotating charged particles close to the light surface in a plasma-rich pulsar magnetosphere and study their interactions with magnetic and photon fields under conditions appropriate for Crab-type pulsars. Methods. Centrifugal acceleration of particles in a monopol-like magnetic field geometry is analyzed and the efficiency constraints, imposed by corotation, inverse Compton interactions and curvature radiation reaction are determined. We derive expressions for the maximum particle energy and provide estimates for the corresponding high-energy curvature and inverse Compton power outputs. Results. It is shown that for Crab-like pulsars, electron Lorentz factor up to $\gamma \sim 10^{7}$ can be achieved, allowing inverse Compton (Klein-Nishina) up-scattering of thermal photons to $\mathrm{TeV}$ energies with a maximum luminosity output of $\sim 10^{31} \mathrm{erg} / \mathrm{s}$. Curvature radiation, on the other hand, will result in a strong $\mathrm{GeV}$ emission output of up to $\sim\left(10^{34}-10^{35}\right) \mathrm{erg} / \mathrm{s}$, quasi-exponentially decreasing towards higher energies for photon energies below $\sim 50 \mathrm{GeV}$.

Conclusions. Accordingly to the results presented only young pulsars are expected to be sites of detectable VHE $\gamma$-ray emission.
\end{abstract}

Key words. stars: pulsars: general - acceleration of particles - radiation mechanisms: non-thermal

\section{Introduction}

One of the fundamental problems in pulsar physics is related to the origin of the observed non-thermal emission. While it seems evident that efficient particle acceleration and emission processes must be operating in a pulsar's rotating magnetosphere, current theoretical approaches differ widely in their assumptions about the localization of the relevant zones: According to standard polar cap models, for example, charged particles are uprooted from the neutron star's surface by strong electrostatic fields (Ruderman \& Sutherland 1975). Still close to the star, these particles are then assumed to be efficiently accelerated along open field lines in parallel electric fields induced by space-charge-limited flow (Michel 1991), field-line curvature (Arons 1983) and/or inertial frame dragging effects (Muslimov \& Tsygan 1992). In most cases, the parallel electric field component is shorted out at some altitude by the onset of electron-positron pair cascades in strong magnetic fields (one-photon pair production), either initiated by curvature (Daugherty \& Harding 1982) or Inverse Compton radiation (Dermer \& Sturner 1994). Outer gap models, on the other hand, assume that primary particles are efficiently accelerated in vacuum gaps in the outer magnetosphere, inducing pair cascades through $\gamma \gamma$-pair production (Cheng et al. 1986; Chiang \& Romani 1994; Hirotani 2007). In all these approaches the maximum attainable particle energy is either limited by the gap size or radiation reaction. In conventional polar cap models, for example, a critical issue has always been the question whether particles can indeed gain enough energy inside the gap to account for the observed non-thermal radiation from $\gamma$-ray pulsars. Several scenarios have been proposed to enlarge the gap zone and consequently increase the corresponding energy output (e.g., Usov \& Shabad 1985; Arons \& Scharlemann 1979; Muslimov \& Tsygan 1992), yet $\gamma$-ray emission from Crab-like pulsars still proves challenging to account for. The efficiency of particle acceleration along magnetic field lines has also been studied more recently based on numerical solutions of the structure of a stationary, axisymmetric and force-free magnetosphere of an aligned pulsar (Contopoulos et al. 1999). According to the results obtained, the relativistic magnetospheric outflow is not accelerated efficiently enough to account for the production of high energy gamma-rays. In some respects, this result may not come unexpected as the magnetic field configuration is restricted to be force-free, thus preventing efficient acceleration. In a rather different approach, Beskin \& Rafikov (2000) analyzed the acceleration of a (stationary) two-component, electron-positron outflow in a monopole magnetic field configuration for high (Michel) magnetization parameters $\sigma \equiv B^{2} / 8 \pi n m_{\mathrm{e}} c^{2}$, indicating the ability of the field to sling particles to high velocities. Here, $n_{\mathrm{e}}$ is the electron density, $B$ the induction of the magnetic field, $c$ is the speed of light and e and $m_{\mathrm{e}}$ are electron's charge and the rest mass respectively. Considering plasma dynamics close to the force-free regime (first-order correction), they showed that for small longitudinal currents very high Lorentz factors can be achieved, with almost all of the electromagnetic energy being 
converted into the kinetic energy of particles $\left(\gamma_{\max } \sim \sigma\right)$ in a thin layer close to the light cylinder surface.

In the present contribution we consider another acceleration mechanism that may help to overcome the energy problem arising in some polar cap-type models. To this end, we explore the acceleration of co-rotating charged particles in an idealized monopole-like magnetic field region close to the light surface where the parallel electric field component is effectively screened out, but where inertial (centrifugal) effects become important in describing the plasma dynamics. This follows earlier suggestions by Gold $(1968 ; 1969)$ about efficient particle acceleration close the light cylinder in a corotating neutron star magnetosphere (see also Ruderman 1972). A detailed analysis of centrifugal acceleration along rotating straight field lines in the test particle limit has been presented by Machabeli \& Rogava (1994), showing that due to the relativistic mass increment the radial acceleration of a particle changes sign, similar to results obtained for particle motion close to a Schwarzschild black hole (Abramowicz \& Prasanna 1990). Based on this, the plasma motion in pulsar magnetospheres has been analyzed and equations describing the behavior of a co-rotating plasma stream have been derived (e.g., Chedia et al. 1996; Machabeli et al. 2005). More recently, the generalization to curved field lines (e.g., Archimedes spiral, where a particle may asymptotically reach the force-free regime) has been examined and the consequences of radiation reaction analyzed (Rogava et al. 2003; Dalakishvili et al. 2007). Independently, applications of centrifugal particle acceleration to milli-second pulsars were considered and curvature radiation effects discussed in Gangadhara (1996) (see also Thomas \& Gangadhara 2007, for a recent generalization). In a wider context, the efficiency of centrifugal particle acceleration was studied for Active Galactic Nuclei (AGN) (Gangadhara \& Lesch 1997; Rieger \& Mannheim 2000; Osmanov et al. 2007), based on scenarios where AGN jets originate as centrifugally-driven outflows (Blandford \& Payne 1982).

In the present paper, we analyze the efficiency of centrifugal acceleration for Crab-like pulsars, taking constraints imposed by co-rotation, inverse Compton interactions and curvature radiation into account. The paper is arranged as follows: In Sect. 2 the radial particle motion due to centrifugal acceleration effects is described and co-rotation constraints discussed. In Sect. 3 we examine possible radiative feedbacks on the process of acceleration for typical millisecond pulsars, considering some major limiting processes: inverse Compton scattering, curvature radiation and pair creation. In Sect. 4 the relevance of our results is shortly discussed in the context of recent observational evidence.

\section{Centrifugal acceleration of particles}

\subsection{A simplified approach}

We consider an idealized, single-particle approach, where in the local frame of reference, each particle is only subject to the action of the centrifugal force and gains energy while moving outward along the magnetic field. The field configuration is supposed to be almost straight, at least inside the co-rotation zone. This seems a reasonable assumption to make as efficient particle acceleration is expected to take place on characteristic length scales much smaller than the light cylinder radius. When applied to open field lines, our results are thus not expected to be very sensitive to further magnetospheric details. If $\alpha$ denotes the angle between the magnetic field $\boldsymbol{B}$ and angular velocity $\boldsymbol{\Omega}$, then the effective field line rotation is $\Omega_{\mathrm{e}}=\Omega \sin \alpha$ and the light surface is at a distance $r_{\mathrm{L}}=r_{\mathrm{L}, 0} / \sin \alpha$, where $r_{\mathrm{L}, 0}=c P / 2 \pi$ is the light cylinder radius and $P=2 \pi / \Omega$ is the pulsar period. The dynamics of a co-rotating particle can then be described by the following equation (Machabeli \& Rogava 1994; Rieger \& Mannheim 2000):

$\frac{\mathrm{d}^{2} r}{\mathrm{~d} t^{2}}=\frac{\Omega_{\mathrm{e}}^{2} r}{1-\Omega_{e}^{2} r^{2}}\left[1-\Omega_{\mathrm{e}}^{2} r^{2}-2\left(\frac{\mathrm{d} r}{\mathrm{~d} t}\right)^{2}\right]$,

and its Lorentz factor can be expressed as

$\gamma(r)=\frac{1}{\sqrt{\tilde{m}}\left(1-r^{2} / r_{\mathrm{L}}^{2}\right)}$,

where $\tilde{m} \simeq 1 / \gamma_{0}^{2}$ is essentially determined by the initial conditions. Hence, for a secondary pair plasma produced close to the neutron star with Lorentz factor $\gamma_{0} \sim\left(10^{3}-10^{4}\right)$, for example, efficient centrifugal acceleration to high energies acceleration can take place close to the light surface, i.e., in a layer $\Delta r / r_{\mathrm{L}} \sim \gamma_{0} / \gamma$. Using Eq. (2), the characteristic timescale for centrifugal acceleration can be approximated by

$t_{\mathrm{acc}} \equiv \frac{\gamma}{\mathrm{d} \gamma / \mathrm{d} t} \simeq \frac{P}{4 \pi \sin \alpha \tilde{m}^{1 / 4} \gamma^{1 / 2}}$

Obviously, for a particle approaching the light surface, the acceleration timescale decreases with $\gamma$ and the Lorentz factor can increase dramatically unless co-rotation can no longer be maintained or radiation reaction becomes important.

\subsection{Co-rotation constraints}

Suppose that the co-rotation zone extends outwards from the neutron star up to the vicinity of the the light surface (Gold 1968, 1969). Because of strong synchrotron losses, electrons will quickly lose their relativistic perpendicular energy, i.e. on a timescale which for most pitch angles $\psi$ is much smaller than the transit time $\tau_{t}=\gamma^{2} \sin ^{2} \psi t_{\mathrm{s}}$ to their ground Landau state, so that they may be approximately described as moving onedimensionally along the field lines. Yet, even if one neglects radiation reaction (e.g., curvature losses, see below) co-rotation will only be possible as long as the kinetic energy density of the electrons $\gamma n m_{\mathrm{e}} c^{2}$ does not exceed the energy density in the field $B^{2} / 8 \pi$ (Alfvén corotation condition). For a number density $n=M n_{\mathrm{GJ}}$, where $M$ denotes the multiplicity (number of secondaries to number of primaries) and $n_{\mathrm{GJ}}=\Omega B \cos \alpha /(2 \pi e c)=$ $0.07 B \cos \alpha / P$ [particles $\mathrm{cm}^{-3}$ ] the classical Goldreich-Julian number density close to the star, the co-rotation condition implies an upper limit for achievable electron Lorentz factors of

$\gamma_{\max , \mathrm{e}}^{\mathrm{cor}} \simeq 4 \times 10^{5} \frac{B P}{M \cos \alpha}$.

For a Crab-type pulsar with $P \sim 0.033 \mathrm{~s}$ and $B \simeq 10^{6} \sin ^{3} \alpha \mathrm{G}$ (at the light surface $r_{\mathrm{L}}$, assuming an internal dipolar field structure), for example, this results in $\gamma_{\text {max,e }}^{\text {cor }} \simeq 2 \times 10^{10} \tan \alpha \sin ^{2} \alpha / M$, while for a $1 \mathrm{~s}$ pulsar $\left(B \sim 10 \sin ^{3} \alpha \mathrm{G}\right.$ at $\left.r_{\mathrm{L}}\right)$ the upper limit would be of the order of $\gamma_{\max , \mathrm{e}}^{\text {cor }} \simeq 4 \times 10^{7} \tan \alpha \sin ^{2} \alpha / M$. These values suggest that for milli-second pulsars co-rotation may indeed last up to the very vicinity of the light cylinder. Note that close to the light surface, the number density required to screen out a potential parallel electric field component is much larger than $n_{\mathrm{GJ}}$ employed above, and in fact given by (Goldreich \& Julian 1969)

$n_{\mathrm{GJ}, \mathrm{L}}^{\mathrm{L}}=n_{\mathrm{GJ}} \frac{1}{\left(1-\Omega_{\mathrm{e}}^{2} r^{2} / c^{2}\right)}$ 
The onset of a pair production front in polar cap models, close to the neutron star, is usually expected to result in a multiplicity $M=n / n_{\mathrm{GJ}} \sim 10^{2}-10^{5}$ and a mean Lorentz factors of the secondaries of $\gamma_{0} \sim 10^{3}-10^{4}$ (e.g., Daugherty \& Harding 1983; Melrose 1998; Baring 2004). On the other hand, using Eqs. (2), (5) can be expressed as $n_{\mathrm{GJ}, \mathrm{L}} \simeq$ $n_{\mathrm{GJ}} \gamma / \gamma_{0}$. Hence, in order to achieve electron Lorentz factors of, e.g., $\gamma \sim 10^{7}$ via centrifugal acceleration, one requires $M \sim \gamma / \gamma_{0} \sim\left(10^{3}-10^{4}\right)$, which seems well possible given the parameter range above. Equation (4) then suggests conversely, that for Crab-type pulsars, Lorentz factors up to $\gamma_{\text {max,e }} \sim 10^{7}$ can indeed be achieved if radiation reaction is negligible. Acceleration will then occur in a narrow region very close to the light surface with $\Delta r / r_{\mathrm{L}} \sim 1 / M$. This seems reminiscent of earlier results about particle acceleration in the presence of small longitudinal currents (Beskin et al. 1983; Beskin \& Rafikov 2000).

\section{Emission constraints}

In realistic astrophysical environments, radiation reaction will impose additional constraints on the efficiency of any particle acceleration process. For pulsars, important limitations could arise through inverse Compton scattering with ambient soft photons field or synchro-curvature losses along curved particle trajectories. Pair production (i.e., one-photon or photon-photon) on the other hand, could possibly lead to a suppression of detectable high energy $\gamma$-rays.

\subsection{Inverse compton interactions}

Thermal radiation as well as synchrotron radiation by secondary electrons could in principle lead to a non-negligible target photon field for Inverse Compton (IC) interactions and thereby limit achievable electron energies.

\subsubsection{Inverse Compton with thermal photons}

It has often been assumed that IC interactions with thermal photons from the neutron star surface are generally negligible far away from the surface because (i) the photon density decreases with distance $r$; and (ii) charges and photon are traveling in almost the same direction, so that (anisotropic) inverse Compton losses become exceedingly small (e.g., Morini 1981). While the first consideration is certainly true, the latter may not necessarily be the case. In fact, if electrons are co-rotating with the plasma, their main velocity component close to the light cylinder is expected to be in the azimuthal direction, implying a preferred interaction angle of almost 90 degree, so that IC interactions with thermal photon field may possibly become relevant for milli-second pulsars. Although pulsars are born at very high temperatures $T \sim 10^{11} \mathrm{~K}$, their surface temperatures quickly cool down to $\sim 5 \times 10^{6} \mathrm{~K}$ by various neutrino emission processes and thermal emission of photons (e.g., Tsuruta et al. 2002; Yakovlev \& Pethick 2004). Standard (modified UCRA, plasma neutrino and photon cooling) models predict a surface temperature above $10^{6} \mathrm{~K}$ for pulsars with ages $\tau=P / 2 \dot{P} \lesssim 10^{4}$ yr (neutrino cooling stage), and below $10^{5} \mathrm{~K}$ for pulsars exceeding $10^{6.7} \mathrm{yr}$ (photon cooling stage). Using the standard cooling curve, one can employ an approximate phenomenological description for the temperature-age dependence given by (Zhang \& Harding 2000)

$T(\tau) \simeq 5.9 \times 10^{5} \mathrm{~K}\left(\frac{10^{6} \mathrm{yr}}{\tau}\right)^{0.1} \quad$ for $\tau \leq 10^{5.2} \mathrm{yr}$,
$T(\tau) \simeq 2.8 \times 10^{5} \mathrm{~K}\left(\frac{10^{6} \mathrm{yr}}{\tau}\right)^{0.5} \quad$ for $\tau>10^{5.2} \mathrm{yr}$

Hence, for a Crab-type pulsar $\left(\tau \simeq 10^{3} \mathrm{yr}, r_{\mathrm{s}} / r_{\mathrm{L}, 0} \simeq 10^{-2}\right)$ (where $r_{\mathrm{s}}$ is the stellar radius) the surface temperature may be approximated by $T \simeq 1.2 \times 10^{6} \mathrm{~K}$. The Planck function then peaks at around $v_{\max }=2.8 \mathrm{kT} / \mathrm{h} \simeq 7 \times 10^{16} \mathrm{~Hz}$, corresponding to a photon energy $\epsilon_{\mathrm{ph}} \simeq 0.3 \mathrm{keV}$. IC scattering thus mainly occurs in the extreme Klein-Nishina regime. As a first order approximation for the single particle (non-resonant) Klein-Nishina Compton power, one can employ the expression derived by Blumenthal \& Gould (1970) assuming a (quasi-isotropic) blackbody photon distribution, i.e.,

$$
P_{\mathrm{c}, \mathrm{KN}} \simeq \frac{\sigma_{T}\left(m_{\mathrm{e}} c k T\right)^{2}}{16 \hbar^{3}}\left(\ln \frac{4 \gamma k T}{m_{\mathrm{e}} c^{2}}-1.981\right)\left(\frac{r_{\mathrm{s}}}{r}\right)^{2},
$$

where $\gamma$ is the electron Lorentz factor. This implies a characteristic IC cooling timescale close to of $r_{\mathrm{L}}$ of

$t_{\mathrm{IC}}=\frac{\gamma m_{\mathrm{e}} c^{2}}{P_{\mathrm{c}, \mathrm{KN}}} \propto \gamma$,

which to first order is proportional to $\gamma$. Acceleration, on the other hand, occurs on a timescale $t_{\text {acc }} \propto 1 / \gamma^{1 / 2}$, so that for electron Lorentz factors $\gamma \gtrsim \gamma_{0}$, IC cooling will not impose any constraints on achievable particle energies. This suggests that for Crab-like pulsars electron Lorentz factors are essentially limited by co-rotation and not by IC radiation reaction (cf. Eq. (4)). If so, then detectable IC emission at $\gamma m_{\mathrm{e}} c^{2} \sim 5\left(\gamma / 10^{7}\right) \mathrm{TeV}$ may occur. We can roughly estimate the possible TeV luminosity by multiplying the corresponding particle number with the single IC power $P_{\mathrm{c}, \mathrm{KN}}$, i.e., $L_{\mathrm{IC}}^{\mathrm{TeV}} \sim n_{\mathrm{GJ}} M \Delta V P_{\mathrm{c}, \mathrm{KN}}$, which gives

$L_{\mathrm{IC}}^{\mathrm{TeV}} \sim 10^{31}\left(\frac{B}{10^{6} \mathrm{G}}\right)\left(\frac{T}{1.2 \times 10^{6} \mathrm{~K}}\right)^{2}\left(\frac{\chi}{\sin \alpha}\right) \mathrm{erg} / \mathrm{s}$,

where $\Delta V=4 \pi \chi r_{\mathrm{L}}^{2} d \sim 2 \pi \chi r_{\mathrm{L}}^{3} / M$, with $\chi \leq 1$ denoting the deviation from isotropy and $d \sim\left(\gamma_{0} / 2 \gamma\right) r_{\mathrm{L}}$ the thickness of the layer close to $r_{\mathrm{L}}$, in which the highest particle energies are achieved. The (pulsed, non-steady) TeV luminosity could thus be as high as $\sim 10^{31} \mathrm{erg} / \mathrm{s}$, consistent with existing upper limits derived by current ground-based $\gamma$-ray instruments (IACT) (e.g., Lessard et al. 2000; Aharonian et al. 2007; Albert et al. 2008), yet possibly accessible to the next-generation CTA-type instruments.

\subsubsection{Inverse Compton with infrared photons}

Secondary synchrotron emission could possibly lead to a nonnegligible photon field in the infrared-optical regime where IC interactions may occur in the Thomson regime. For the Crab pulsar, the (isotropic, phase-averaged) near infrared-optical luminosity is of order $L_{\mathrm{o}} \sim 10^{33} \mathrm{erg} / \mathrm{s}$, turning significantly downward for lower frequencies (e.g., Middleditch et al. 1983; Eikenberry et al. 1997; Sollerman 2003). This suggests a photon energy density close to the light surface of order $u_{\mathrm{ph}} \sim$ $L_{\mathrm{o}} /\left(4 \pi r_{\mathrm{L}} c\right) \sim 10^{5} \sin ^{2} \alpha \mathrm{erg} / \mathrm{cm}^{3}$, comparable to the thermal one. Approximating the single particle (non-resonant, quasiisotropic) Compton power by $P_{\mathrm{c}} \sim \sigma_{T} c \gamma^{2} u_{\mathrm{ph}} /(1+x)$, where $x=\gamma \epsilon_{\mathrm{ph}} / m_{\mathrm{e}} c^{2}$, the characteristic IC cooling timescale close to $r_{\mathrm{L}}$ then becomes

$t_{\mathrm{IC}} \simeq \frac{\gamma m_{\mathrm{e}} c^{2}}{P_{\mathrm{c}}} \simeq \frac{4.1 \times 10^{7} \mathrm{~s}}{\gamma u_{\mathrm{ph}, \mathrm{L}}}\left(1+2 \gamma / 10^{6}\right)$. 
Comparing acceleration, occurring on $t_{\text {acc }}$ (Eq. (3)), with IC cooling, occurring on $t_{\mathrm{IC}}$, implies

$$
\frac{\gamma}{\left(1+2 \gamma / 10^{6}\right)^{2}} \lesssim \frac{2 \times 10^{10}}{\gamma_{0} \sin ^{2} \alpha}
$$

and verifies that IC interactions with the infrared-optical photon field will not impose a severe constraint on the maximum achievable Lorentz factor. IC up-scattering by electrons with $\gamma \sim 10^{7}$ would again produce emission at around $5 \mathrm{TeV}$. If the observed NIR-optical emission would originate from regions close to the pulsar, IC scattering close to $r_{\mathrm{L}}$ might be reasonably approximated by the quasi-isotropic expression $P_{\mathrm{c}}$. This would result in a possible TeV output $L_{\mathrm{IC}} \sim$ $n_{\mathrm{GJ}} M \Delta V P_{\mathrm{c}} \sim 10^{35} \chi \sin \alpha \cos \alpha \mathrm{erg} / \mathrm{s}$, exceeding the existing upper limits noted above. On the other hand, if the NIRoptical emission is produced close to $r_{\mathrm{L}}$ as proposed in some models (e.g., Pacini \& Salvat 1983; Crusius-Wätzel et al. 2001), anisotropic inverse Compton scattering may severely reduce this power output (by up to a factor $\psi^{4}$ where $\psi \ll 1$ is the pitch angle, cf. Morini 1981), suggesting a TeV contribution well below the one produced by thermal IC. The empirical scaling of the near infrared-optical (and $\gamma$-ray) flux with the magnetic field at the light cylinder seems in fact to reinforce a scenario where the emitting region is located close to the light surface (Shearer \& Golden 2001).

\subsection{Curvature radiation}

Approaching the light surface, field line bending may no longer be negligible so that a particle may efficiently lose energy due to curvature radiation. In analogy to synchrotron radiation, curvature radiation can be described as emission from relativistic charged particles moving around the arc of a circle, chosen such that the actual acceleration corresponds to the centripetal one (e.g., Ochelkov \& Usov 1980). The critical frequency where most of the radiation is emitted is given by

$v_{\mathrm{c}} \simeq \frac{3 c}{4 \pi R_{\mathrm{c}}} \gamma^{3}$,

which for, e.g., $R_{\mathrm{c}} \sim r_{\mathrm{L}} \sim 10^{8} \mathrm{~cm}$ (cf. Gold 1968, 1969) yields $v_{\mathrm{c}} \simeq 5 \times 10^{22}\left(\gamma / 10^{7}\right)^{3} \mathrm{~Hz}$ or a curvature photon energy of about $0.2\left(\gamma / 10^{7}\right)^{3} \mathrm{GeV}$. The energy loss rate or total power radiated away by a single particle is

$P_{\mathrm{c}}=\frac{2}{3} \frac{e^{2} c}{R_{\mathrm{c}}^{2}} \gamma^{4}$

The characteristic cooling timescale $t_{\mathrm{c}}=\gamma m_{0} c^{2} / P_{\mathrm{c}}$ thus becomes

$t_{\mathrm{c}} \simeq 180 R_{\mathrm{c}}^{2}\left(\frac{m_{0}}{m_{\mathrm{e}}}\right) \frac{1}{\gamma^{3}}$.

To find the maximum electron Lorentz factor attainable in the presence of curvature radiation, we can again balance $t_{\mathrm{acc}}$ (Eq. (3)) with $t_{\mathrm{c}}$ to obtain

$\gamma_{\max } \simeq 1.2 \times 10^{9} P^{2 / 5}\left(\frac{R_{\mathrm{c}}}{r_{\mathrm{L}}}\right)^{4 / 5} \frac{1}{\gamma_{0}^{1 / 5}} \sin ^{2 / 5} \alpha$,

indicating that for a Crab-type pulsar $\left(P=0.033 \mathrm{~s}, R_{\mathrm{c}} \sim r_{\mathrm{L}}\right)$ with, e.g., $\gamma_{0} \sim 10^{4}$ curvature radiation constrains achievable electron Lorentz factors to $\gamma \lesssim 4.8 \times 10^{7} \sin ^{2 / 5} \alpha$. This confirms that for Crab-type parameters, electron Lorentz factors up to $\sim 10^{7}$ might in fact be obtained. We can again estimate the possible curvature output $L_{\mathrm{c}}^{\mathrm{GeV}}$ at $\mathrm{GeV}$ energies, by multiplying the particle number with the single particle curvature power $P_{\mathrm{c}}$ to obtain

$$
\begin{aligned}
L_{\mathrm{c}}^{\mathrm{GeV}} \sim & n_{\mathrm{GJ}} M \Delta V P_{\mathrm{c}} \sim 10^{34}\left(\frac{B}{10^{6} \mathrm{G}}\right)\left(\frac{r_{\mathrm{L}, 0}}{R_{\mathrm{c}}}\right)^{2}\left(\frac{\gamma}{10^{7}}\right)^{4} \\
& \times \frac{\chi \cot \alpha}{\sin ^{2} \alpha} \mathrm{erg} / \mathrm{s}
\end{aligned}
$$

using $\Delta V$ (with $\chi \leq 1)$ as defined above. Note that curvature radiation in principle results in an additional IC (Klein-Nishina) photon target field close to $r_{\mathrm{L}}$ with energy density $u_{\mathrm{ph}, \mathrm{c}} \sim$ $L_{\mathrm{c}}^{\mathrm{GeV}} /\left(4 \pi r_{\mathrm{L}}^{2} c\right)$. For Crab-type values, the resultant energy density would be a factor of a few higher than the thermal one. Yet, due to both, the further reduced Klein-Nishina cross-section and anisotropic scattering conditions, this curvature photon field is not expected to significantly modify our IC considerations above.

\subsection{Pair creation and $\gamma \gamma$-absorption}

In the magnetosphere of a pulsar, pair creation via magnetic photon absorption $\left(\gamma+\boldsymbol{B} \rightarrow e^{+}+e^{-}+\boldsymbol{B}\right)$ becomes kinematically possible as the magnetic field can absorb momentum and thereby ensure momentum conservation. For a photon, traveling with pitch angle $\psi$ to the local magnetic field, the threshold conditions for this being possible is $\epsilon_{\mathrm{ph}} \sin \psi \geq$ $2 m_{\mathrm{e}} c^{2}$. The absorption coefficient for this process is $\kappa \simeq 1.4 \times$ $10^{-13}\left(B \sin \psi / B_{\mathrm{c}}\right) T(\lambda)[1 / \mathrm{cm}]$ where $B_{\mathrm{c}}=m_{\mathrm{e}} c^{3} /(e \hbar) \simeq 4.4 \times$ $10^{13} \mathrm{G}, \lambda=1.5\left(B \sin \psi / B_{\mathrm{c}}\right)\left(h v / m_{\mathrm{e}} c^{2}\right)$ and $T(\lambda)$ is the Erber function (Erber 1966; Tsai \& Erber 1974). The function $T(\lambda)$ is very sensitive to $\lambda$. While $\lambda$ might be high near to the stellar surface, only moderate values are expected close to the light surface. For small $\lambda \ll 1$ one has $T(\lambda) \simeq 0.46 \exp (-4 / \lambda)$ (cf. also Daugherty \& Harding 1983), while for large $\lambda \gg 1$ one finds $T(\lambda) \simeq 0.9 \lambda^{-1 / 3}$. The location of the absorbing surface may thus be approximated by $\kappa r \simeq 1$, which gives

$\lambda^{-1} \simeq-0.25 \ln \left[6.4 \times 10^{-14}\left(B / B_{\mathrm{c}}\right) r\right]$.

For a Crab-type pulsar $\left(B \sim 10^{6} \sin ^{3} \alpha \mathrm{G}\right.$ close to $\left.r_{\mathrm{L}}\right)$ one finds $\lambda^{-1} \simeq 7.6$, so that a photon with energy above the cut-off $\epsilon_{\mathrm{ph}}=h v \sim 2 /\left(\sin ^{3} \alpha \sin \psi\right) \mathrm{TeV}$ will undergo magnetic absorption. As the pitch angle is usually very small $\left(\psi \lesssim 10^{-2}\right)$, we do not expect this of significance for Crab-type pulsars at photon energies below $50 \mathrm{TeV}$.

Apart from one-photon pair production, energetic photons may also undergo photon-photon interactions $\left(\gamma+\gamma_{\mathrm{s}} \rightarrow\right.$ $\left.e^{+}+e^{-}\right)$with background soft photons of energy $\epsilon_{\mathrm{s}}$ (e.g., Chiang \& Romani 1994). Let us thus consider the following cases:

(1) In the case of $\mathrm{TeV}$ photons, the threshold condition requires the presence of soft photons with energies $\epsilon_{\mathrm{s}}=2\left(m_{\mathrm{e}} c^{2}\right)^{2} / \epsilon=$ $0.5(1 \mathrm{TeV} / \epsilon) \mathrm{eV}$ or larger. The cross-section for $\gamma \gamma$-pair production has a sharp maximum of $\sim 0.2 \sigma_{T}$ at $2 \epsilon_{\mathrm{s}}$, so that the optical depth can be approximated by $\tau(\epsilon) \sim 0.1 \sigma_{T} L\left(2 \epsilon_{\mathrm{S}}\right) \Delta r /\left(4 \pi r^{2} c \epsilon_{\mathrm{S}}\right)$ where $L\left(2 \epsilon_{\mathrm{S}}\right)$ is the corresponding luminosity at which the peak occurs and $\Delta r \simeq\left(r-r_{\mathrm{L}}\right)$ the path length. For a characteristic (observed pulsed Crab) photon field of $L(0.1 \mathrm{eV}) \ll L(1 \mathrm{eV}) \lesssim$ $(1-3) \times 10^{33} \mathrm{erg} / \mathrm{s}$ (e.g., Eikenberry et al. 1997; Sollerman 2003) this would result in

$\tau(\epsilon) \simeq 1\left(\frac{L(1 \mathrm{eV}[1 \mathrm{TeV} / \epsilon])}{3 \times 10^{33} \mathrm{erg} / \mathrm{s}}\right)\left(\frac{\epsilon}{1 \mathrm{TeV}}\right) \sin \alpha$ 
noting that $\left(r_{\mathrm{L}} / r\right)\left(1-r_{\mathrm{L}} / r\right) \leq 1 / 4$ for $r \geq r_{\mathrm{L}}$. Equation (19) thus suggests that a substantial fraction of $\mathrm{TeV}$ photons (if not all) may in fact be able to escape absorption.

(2) The situation could be somewhat different for the GeV curvature photons. In this case $\gamma \gamma$-absorption requires the presence of soft photons in the X-ray regime with $\epsilon_{\mathrm{s}}=0.5(1 \mathrm{GeV} / \epsilon)$ $\mathrm{keV}$ or larger. In the case of, e.g., the Crab pulsar, the X-ray luminosity is of order $L_{x} \sim 10^{36} \mathrm{erg} / \mathrm{s}$ (Kuiper et al. 2001; Possenti et al. 2002; Massaro et al. 2006), so that the optical depth becomes

$\tau(\epsilon) \simeq 0.34\left(\frac{L(1 \mathrm{keV}[1 \mathrm{GeV} / \epsilon])}{10^{36} \mathrm{erg} / \mathrm{s}}\right)\left(\frac{\epsilon}{1 \mathrm{GeV}}\right) \sin \alpha$.

To first order, the soft X-ray flux of the Crab follows $L(E) \simeq 10^{36}(10 \mathrm{keV} / E)^{-0.4} \mathrm{erg} / \mathrm{s}$ (e.g., Massaro et al. 2000; Kuiper et al. 2001). Hence, for photons of energy $\epsilon \sim 20 \mathrm{GeV}$ or $50 \mathrm{GeV}$, the relevant target luminosity would be $L\left(2 \epsilon_{\mathrm{s}}\right) \simeq$ $1.2 \times 10^{35}$ and $\simeq 8.3 \times 10^{34} \mathrm{erg} / \mathrm{s}$ respectively, implying an optical depth $\tau \simeq 0.8 \sin \alpha$ and $\tau \simeq 1.4 \sin \alpha$. As curvature radiation typically results in a quasi-exponential $\mathrm{GeV}$ tail, no significant super-exponential suppression is expected to occur at energies below several tens of $\mathrm{GeV}$, which seems consistent with recent results based on the detection of pulsed emission above $25 \mathrm{GeV}$ from the Crab (Aliu et al. 2008).

\section{Conclusions}

Based on our idealized model analysis above, the efficiency of particle acceleration close to the light surface is essentially limited by curvature radiation reaction or the Alfvén corotation condition.

We have studied the implications for conditions applicable to Crab-type pulsars, assuming a plasma-rich environment with pair density exceeding the primary Goldreich-Julian (close to the star) one by $M \sim 10^{3}$. In this case, electron Lorentz factors up to $\gamma \sim 2 \times 10^{7}$ appear possible. Synchro-curvature radiation could then lead to a relatively strong (averaged) power output of $\sim\left(10^{34}-10^{35}\right) \mathrm{erg} / \mathrm{s}$ at $\sim 2 \mathrm{GeV}$ that would be consistent with, e.g., EGRET observations of the Crab (Kuiper et al. 2001). The emissivity of curvature radiation can be described by

$p(v)=\frac{1}{2} \sqrt{\frac{3}{\pi}} \frac{\mathrm{e}^{2}}{R_{\mathrm{c}}} \gamma \frac{v}{v_{\mathrm{c}}} \int_{v / v_{\mathrm{c}}}^{\infty} \frac{\mathrm{e}^{-x}}{\sqrt{x}} \mathrm{~d} x$.

For a power law-type distribution of particles, for example, $N(\gamma)=N_{0} \gamma^{-s}$, one can show that for high frequencies $\left(v \gg v_{\mathrm{c}}\right)$ the intensity $I_{v}$ behaves as

$I_{v} \propto \int p(v, \gamma) N(\gamma) \mathrm{d} \gamma \propto \mathrm{e}^{-v / v_{\mathrm{c}}}$,

so that in the case of the Crab the curvature output might be expected to decay quasi-exponentially for energies above $\sim 10 \mathrm{GeV}$, as indeed suggested by recent very high energy gamma-ray measurements (Albert et al. 2008; Aliu et al. 2008). Inverse Compton (Klein-Nishina) up-scattering of thermal soft photons, on the other hand, could result in a (quasi-isotropic) power output of $\sim 10^{31} \mathrm{erg} / \mathrm{s}$ at $\mathrm{TeV}$ energies, consistent with, e.g., existing IACT constraints on the observed (non-steady) emission from the Crab.

For older pulsars (e.g., $P \sim 1 \mathrm{~s}$ ) and $M \gg 1$, co-rotation usually imposes the strongest constraint, so that achievable maximum Lorentz factors are typically limited to $\sim\left(10^{4}-10^{5}\right)$. Although curvature radiation may then peak in the optical-UV

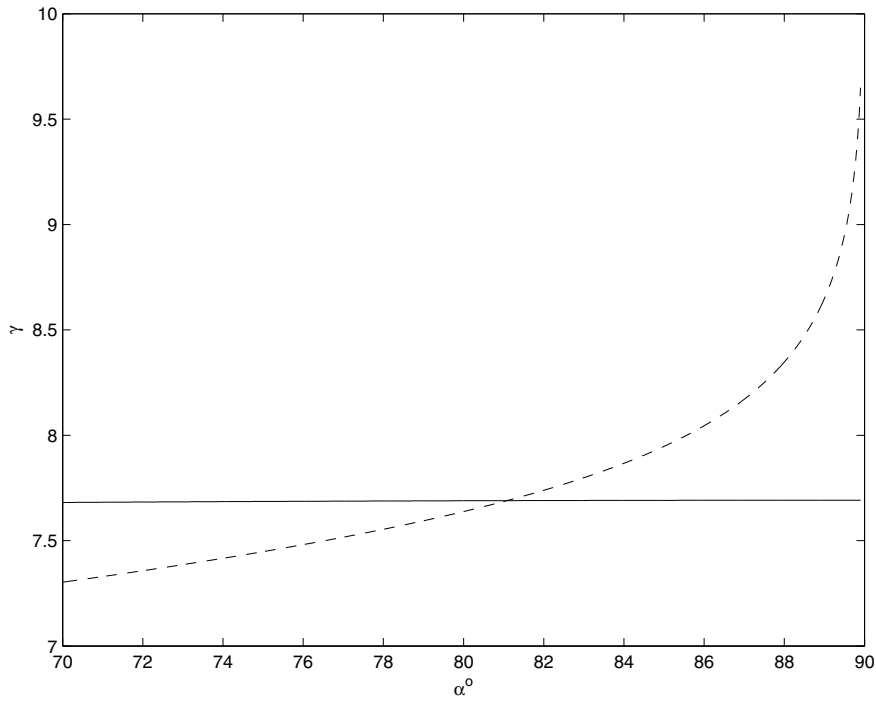

Fig. 1. Maximum Lorentz factors versus the inclination angle: $\gamma_{\max }^{\text {cor }}$ (from co-rotation) and $\gamma_{\max }^{\mathrm{cr}}$ (curvature radiation) are represented by the dashed and the solid line respectively. The set of parameters emplyed is: $M=5 \times 10^{3}, P=0.033 \mathrm{~s}, \gamma_{0}=10^{4}$.

(up to $\sim 10 \mathrm{eV}$ ) and inverse Compton (Thomson) scattering of curvature or thermal photons could result in very high energy emission up to $\sim 50 \mathrm{GeV}$, their associated power is negligible due to the small electron Lorentz factors and the substantially reduced target photon energy density. Hence, within the approach considered only young pulsars $(P \lessgtr 0.1 \mathrm{~s})$ might be expected to produce detectable high energy gamma-ray emission.

The proposed scenario could in principle work for a variety of angles, so that the resultant emission needs not necessarily to be strongly pulsed. Note that for most circumstances, the major condition limiting the Lorentz factors of electrons results from co-rotation. Yet, for large inclination angles, curvature radiation reaction can become dominant over co-rotation, cf. Eqs. (4) and (16) and see Fig. 1 for illustration.

The analysis presented is based on a number of idealizations, which we plan to remedy in future studies. This particularly involves the assumptions of, e.g., quasi-straight field lines and a single particle approach in which plasma effects are neglected. On the other hand, one of the strengths of the present concept is its ability to explicitly take inertial effects into account and so to allow to estimate the size and extent of the VHE regions in young pulsars.

Acknowledgements. Discussions with Felix Aharonian, George Machabeli and Vasily Beskin are gratefully acknowledged. Z.O. acknowledges the hospitality of the Max-Plank Institute for Nuclear Physics (Heidelberg, Germany) during his short term visits. The study of Z.O. was partially supported by the Georgian National Science Foundation grant GNSF/ST06/4-096.

\section{References}

Aharonian, F., Akhperjanian, A. G., Bazer-Bachi, A. R., et al. (HESS Collaboration) 2007, A\&A, 466, 543

Abramowicz, M. A., \& Prasanna, A. R. 1990, MNRAS, 245, 729

Albert J., Aliu, E., Anderhub, H., et al. (MAGIC Collaboration) 2008, ApJ, 674, 1037

Aliu, E., et al. (MAGIC Collaboration) 2008, Science, 322, 1224

Arons, J. 1983, ApJ 266, 215

Arons, J., \& Scharlemann, E. T. 1979, ApJ, 231, 854

Beskin, V. S., Gurevich, A. V., \& Istomin, Ya. N. 1983, Sov. Phys. JETP, 58, 235 
Beskin, V. S., \& Rafikov, R. R. 2000, MNRAS, 313, 433

Blandford, R. D., \& Payne, D. G. 1982, MNRAS, 199, 883

Blumenthal, G. R., \& Gould, R. J. 1970, Rev. Mod. Phys., 42, 237

Cheng, K. S., Ho, C., \& Ruderman, M. A. 1986, ApJ, 300, 500

Chedia, O. V., Kahniashvili, T. A., Machabeli, G. Z., \& Nanobashvili, I. S. 1996, Ap\&SS, 239, 57

Chiang, J., \& Romani, R. W. 1994, ApJ, 436, 754

Contopoulos, I., Kazanas, D., \& Fendt, C. 1999, ApJ, 511, 351

Crusius-Wätzel, A. R., Kunzl, T., Lesch, H., et al. 2001, ApJ, 546, 401

Dalakishvili, G. T., Rogava, A. D., \& Berezhiani, V. I. 2007, Phys. Rev. D, 76, 045003

Daugherty, J. K., \& Harding, A. K. 1982, ApJ, 252, 337

Daugherty, J. K., \& Harding, A. K. 1983, ApJ 273, 761

Dermer, C. D., \& Sturner, S. J. 1994, ApJ, 420, L75

Eikenberry, S. S., Fazio, G. G., Ransom, S. M., et al. 1997, ApJ 477, 465

Erber, T. 1966, Rev. Mod. Phys., 38, 626

Gangadhara, R. T. 1996, A\&A, 314, 853

Gangadhara, R. T., \& Lesch, H. 1997, A\&A, 323, L45

Gold, T. 1968, Nature, 218, 731

Gold, T. 1969, Nature, 221, 25

Goldreich, P., \& Julian, W. H. 1969, ApJ, 157, 869

Hirotani, K. 2007, ApJ 662, 1173

Kuiper, L., Hermsen, W., Cusumano, G., et al. 2001, A\&A, 378, 918

Lessard, R. W., Bond, I. H., Bradbury, S. M., et al. (Whipple Collaboration) 2000, ApJ, 531, 942

Lyne, A. G., \& Graham-Smith, F. 2006, Pulsar Astronomy (3rd ed.) (Cambridge Univ. Press)

Machabeli, G. Z., \& Rogava, A. D. 1994, Phys. Rev. A, 50, 98
Machabeli, G. Z., Osmanov, Z. N., \& Mahajan, S. M. 2005, Physics of Plasmas 12,062901

Massaro, E., Cusumano, G., Litterio, M., \& Mineo, T. 2000, A\&A, 361, 695

Massaro, E., Campana, R., Cusumano, G., Mineo, T., et al. 2006, A\&A, 459, 859

Melrose, D. B. 1998, Proc. of APPTC'97 (Toki, Japan), ed. Y. Tomita, et al., 96

Michel, F. C. 1991, Theory of Neutron Star Magnetospheres (Univ. of Chicago Press)

Middleditch, J., Pennypacker, C., \& Burns, M. S. 1983, ApJ, 273, 261

Morini, M. 1981, Ap\&SS, 79, 203

Muslimov, A. G., \& Tsygan, A. I. 1992, MNRAS, 255, 61

Ochelkov, Yu. P., \& Usov, V. V. 1980, Ap\&SS, 69, 439

Osmanov, Z., Rogava, A. S., \& Bodo, G. 2007, A\&A, 470, 395

Pacini, F., \& Salvat, M. 1983, ApJ, 274, 369

Possenti, A., Cerutti, R., Colpi, M., \& Mereghetti, S. 2002, A\&A, 387, 993

Rieger, F. M., \& Mannheim, K. 2000, A\&A, 353, 473

Rogava, A. D., Dalakishvili, G., \& Osmanov, Z. 2003, Gen. Rel. and Grav., 35, 1133

Ruderman, A., \& Sutherland, P. G. 1975, ApJ, 196, 51

Ruderman, M. 1972, ARA\&A 10, 427

Shearer, A., \& Golden, A. 2001, ApJ, 547, 967

Sollerman, J. 2003, A\&A, 406, 639

Tsai, W.-Y., \& Erber, T. 1974, Phys. Rev. D, 10, 492

Thomas, R. M. C., \& Gangadhara, R. T. 2007, A\&A, 467, 911

Tsuruta, S., Teter, M. A., Takatsuka, T., Tatsumi, T., \& Tamagaki, R. 2002, ApJ, 571, L143

Usov, V. V., \& Shabad, A. 1985, Ap\&SS, 117, 309

Yakovlev, D. G., \& Pethick, C. J. 2004, ARA\&A, 42, 169

Zhang, B., \& Harding, A. K. 2000, ApJ, 532, 1150 